\title{
An Efficient Algorithm of the Planar 3-Center Problem for a set of the convex position points
}

\author{
Donglai Bian ${ }^{1}$, Bo Jiang ${ }^{1, \mathrm{a}}$ and Zhiying Cao ${ }^{1}$ \\ ${ }^{1}$ School of Information Science and Technology, Dalian Maritime University, Linghai Road 1, Dalian, 116026, China.
}

\begin{abstract}
The planar 3-center problem for a set $S$ of points given in the plane asks for three congruent circular disks with the minimum radius, whose union can cover all points of $S$ completely. In this paper, we present an $O\left(n^{2} \log ^{3} n\right)$ time algorithm for a restricted planar 3-center problem in which the given points are in the convex positions, i.e. The given points are the vertices of a convex polygon exactly.
\end{abstract}

\section{Introduction}

Let $S$ denote a set of $n$ points given in the plane. The planar $k$-center problem asks for $k$ congruent closed disks of the minimum radius, whose union covers $S$ completely. The problem is NP-complete if $k$ is part of input [1], and can be solved in $O\left(n^{2 k-1} \log n\right)$ for any fixed $k$ [2]. On the other hand, we know that the problem can be solved in $O(n)$ time[3] when $k=1$, i.e. the 1-center problem, and some efficient algorithms are known for $k$ $=2$.

The planar 2-center problem has been studied widely. Sharir studied this problem and proposed a near-linear algorithm with running time $O\left(n \log ^{9} n\right)$ firstly[4]. Later, Eppstein and Chan made some further refinement of Sharir's work, and Eppstein presented a randomized algorithm with $O\left(n \log ^{2} n\right)$ expected time[5], Chan gave out an $O\left(n \log ^{2} n\right)$ time randomized algorithm with high probability, and an $O\left(n(\log n \log \log n)^{2}\right)$ time deterministic algorithm[6]. Kim and Shin have also studied the 2 -center problem for a set of the given points in convex position, and announced an $O\left(n \log ^{3} n \log \log n\right)$ algorithm for covering the whole polygon which is formed by these given points as a vertex, and an $O(n$ $\log ^{2} n$ ) time algorithm for covering all vertices of the polygon respectively[8]. Note that, a set of points is said to be in convex position if it is the set of vertices of a convex polygon. Let $P$ be a convex polygon of $n$ vertices. We also want to find two congruent closed disks of the minimum radius, whose union covers the whole polygon $P$, or only the vertices of $P$. Recently, Tan et al. improved the work of Kim, and presented an $O\left(n \log ^{2} n\right)$ time algorithm which uses the binary search and the known algorithms only, instead of using the complicated parametric search which is the base for most planar 2center algorithms, for computing the smallest enclosing disk of a point set to solve the planar 2-center problem[9].

Although the $k$-center problem originates from the site selection problem, it has some important applications in many areas, such as the modern wireless communication systems, the optimization of modern logistics center, and so on. It is a natural requirement that some transmissions should be set up so that mobile phones in a region can be serviced. For the modern logistics center, some agency shops may be needed. Usually, the service region can be represented by a closed disk, and we can imitate the solution of $k$-center problem and design the optimal strategy for these application problems.

Our work. In this paper, we gave an $O\left(n^{2} \log ^{3} n\right)$ time algorithm for solving a restricted planar 3-center problem in which the given points are in convex position, i.e. these points are the vertices of a minimum convex polygon which surrounds all of the given points.

\section{Preliminaries and notation}

Let $P$ be a convex polygon in the plane and $S$ be a set of vertices of $P$. A convex polygon means that if any edge of it is extended to a straight line in both directions indefinitely, so that all of the other edges should be in the same side of the line. Denote by $S C(P)$ the minimum cover circle of $P$. Denote by $r(R)$ the radius length of circle $R$. Let us number the points on $P$ clockwise by 0 , $1, \ldots, n-1$. Firstly, we sort the vertices of the polygon $P$ in ascending order of the $\mathrm{X}$-axis coordinate size, we mark the first vertex as 0 , and then mark the other vertices as $1,2, \ldots, n-1$ once in a counterclockwise. For two vertices $x$ and $y$ of $P$, denote by $P[x, y]$ the polygonal chain of $P$ from $x$ to $y$ in counterclockwise. Denote by $<x$, $y>$ the point set which are composed of the vertices of

* Corresponding author: ${ }^{a}$ Bo Jiang: bojiang@ dlmu.edu.cn 
$P[x, y]$. Denote by the $A N S \_k(S)$ the answer of the $k$ center problem for $S$.

Lemma 1 For any circle $R$, if the straight line segment $\overline{A B}$ is a chord which does not pass through the center of the circle $R$, then there exists a circle $R^{\prime}$ which covers the area surrounded by the inferior arc $\widehat{A B}$ and the chord $\overline{A B}$, and $r\left(R^{\prime}\right)<r(R)$.

Proof. As shown in Figure $1, \overline{A B}$ is a chord that does not pass through the center of the circle in $R$, and $\overline{C D}$ is the diameter which is parallel to $\overline{A B}$ in the circle $R$. Assume that the point $O$ is the center of $R$, we make a line segment which pass the point $O$ and perpendicular to $\overline{C D}$, intersect line $\overline{A B}$ at point $E$, and circle $R$ at point $F$.

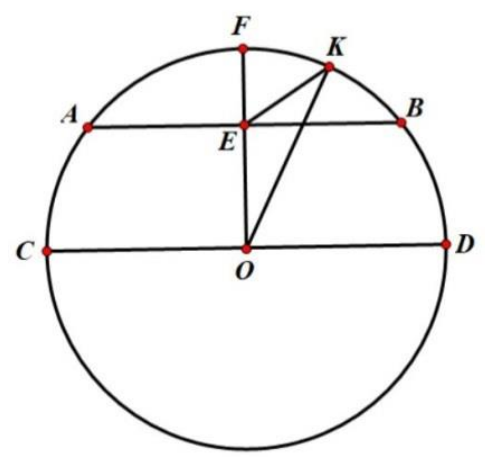

Figure 1. Illustrating for Lemma 1

Suppose $K$ is a point on the inferior arc $\widehat{F B}$, according to the assumption, we have $0 \leq \angle \mathrm{EOK}<\pi / 2, \cos (\mathrm{x})$ is a monotonically decreasing function, when $x \in[0, \pi / 2]$.Thus, when point $K$ coincides with point $B,|E K|$ takes the maximum. When point $K$ coincides with point $F,|E K|$ takes the minimum. The following relationships are established

$$
\overrightarrow{E K}=\overrightarrow{O K}-\overrightarrow{O E}
$$

(1)

$$
\|\overrightarrow{E K}\|=\sqrt{\|\overrightarrow{O K}\|^{2}+\|\overrightarrow{O E}\|^{2}-2\|\overrightarrow{O K}\|\|\overrightarrow{O E}\| \cos \angle E O K}
$$

$$
\|\overrightarrow{E F}\| \leq\|\overrightarrow{E K}\| \leq\|\overrightarrow{E B}\|
$$

By symmetry, the inferior arc $\widehat{A F}$ has the same property. We take point $E$ as the center of circle $R^{\prime}$, and take $\overline{E B}$ as the radius. From the previous discussion, the circle $R^{\prime}$ can cover the area surrounded by the inferior arc $\widehat{A B}$ and the chord $\overline{A B} \cdot \overline{A B}$ is a chord that does not pass through the center of the circle $R$, thus, $|A B|<|C D|$, and $r\left(R^{\prime}\right)<r(R)$.

Lemma 2 Suppose $P$ is a convex polygon in the plane. There exist two congruent circles of the minimum radius, whose union can cover all of the vertices of $P$, and both of the circles are smaller than $S C(P)$.
Proof. For lemma 2, there are three possible conditions for the vertices of diameter of the $S C(P)$ and the vertices of $P$ (There is no common point or one common point or two common points), three cases are discussed as follows.

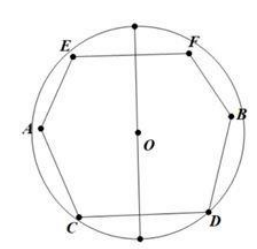

(a)

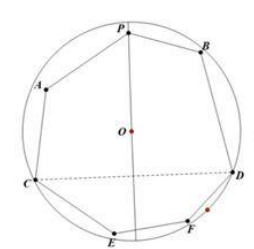

(b)

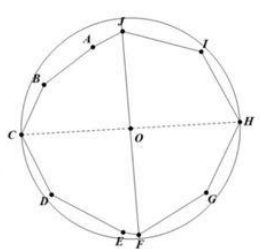

(c)
Figure 2. Illustrating for Lemma 2

Case 1. As shown in Figure 2(a), suppose the polygon $P$ has two points(or more), e.g. Point $C$ and $D$, on the circle $S C(P)$, there is a chord which is perpendicular to the straight line $\overline{C D}$.We make a chord through the point of $E$ and point $C$. We make another string which passes point $F$ and point $D$, according to lemma 1, we can make two circles whose radius is smaller than the radius of the circle $S C(P)$.

Case 2. As shown in Figure 2(b), suppose the polygon $P$ has two points(or more), e.g. Point $C$ and $D$, on the circle $S C(P)$, there is a chord which is perpendicular to the straight line $\overline{C D}$ intersect the polygon $P$ at point $p$. We make a chord through the point of $E$ and point $P$. We make another chord which passes point $F$ and point $B$, according to lemma 1, we can make two circles whose radius is smaller than the radius of circle $S C(P)$.

Case 3. As shown in Figure.2(c), suppose the polygon $P$ has two points (or more)e.g. Point $C$ and $H$, on the circle $S C(P)$, there is a chord which is perpendicular to the straight line $\overline{C H}$ intersect the polygon $P$ at point $J$ and $F$. We make a chord through the point of $F$ and point $A$. We make another chord which passes point $J$ and point $G$, according to lemma 1 ,we can make two circles whose radius is smaller than the radius of circle $S C(P)$.

In summary, lemma 4 is hold.

Inference $1 P$ is the convex polygon in the plane, and the $S$ is a set of vertices of $P$. We have $A N S \_k(S)<$ $r(S C(P))$.

\section{Construction of the minimum radius disk}

Lemma $3 A, B$ are two point sets and $A$ is a subset of $B$, then $A N S \_k(A) \leq A N S \_k(B)$.

Proof. Assume $A N S \_k(A)>A N S \_k(B)$. According to the definition, $A N S \_k(B)$ also can over all points in set $A$, then $A N S \_k(A)$ is not an optimal solution, which is contradictory. Thus, $A N S \_k(A) \leq A N S \_k(B)$.

Observation $1 P$ is a convex polygon on the plane, point $a$ and $b$ are vertices that do not contiguous on $P$. We split the $P$ into two polygons $S U A, S U B$ with the 
straight line $\overline{a b}$, then, both $S U A$ and $S U B$ are convex polygons.

$P$ is a convex polygon which has $n$ vertices has $\frac{n(n-3)}{z}$

ways to split it. Using $S U A_{k}$ and $S U B_{k}$ to represent the two point set of two convex polygons which are split in the $k$-th mode. For example, as shown in Figure 3, $S U A_{k}$ is composed of the vertices of the convex chain $P[i, j]$, and $S U B_{k}$ is composed of the vertices of the convex chain $P[j+1, i-1]$ and a straight line.(The dotted line is an imaginary line).

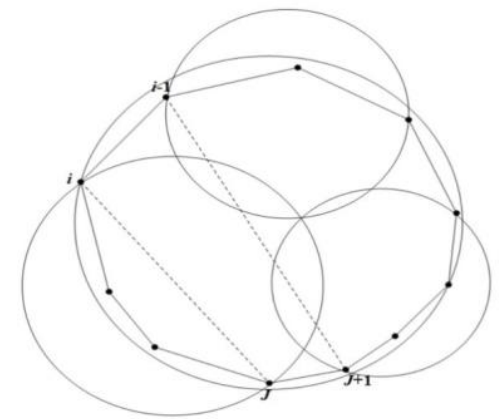

Figure 3. A kind of segmentation

For a number $i \quad(1 \leq i \leq n-1)$, let $\operatorname{br}(<i, j\rangle)=\max \left\{A N S \_1(<i, j-1>), A N S \_2(<j, i-1>)\right\} \quad$ and $\operatorname{gbr}(i)=\{\quad \operatorname{br}(<i, i+1>), \operatorname{br}(<i, i+2>), \ldots \operatorname{br}(<i$, end $>)\}$, end $=(i-2+n) \bmod n$.

\section{Lemma 4}

$A N S \_3(P)=\min \left(\max \left(A N S \_1\left(S U A_{\mathrm{k}}\right), A N S \_2\left(S U B_{\mathrm{k}}\right)\right)\right), 1$ $\leq k \leq \frac{n(n-3)}{2}$

Proof. Let $t r=$ $\min \left(\max \left(A N S \_1\left(S U A_{\mathrm{k}}\right), A N S \_2\left(S U B_{\mathrm{k}}\right)\right)\right)$, $1 \leq k \leq \frac{n(n-3)}{2}$.

Obviously, $A N S \_3(P) \leq t r$. Assume $t r>A N S \_3(P)$, thus $A N S \_1\left(S U A_{k}\right)^{>} A N S \_3(P)$ or $A N S \_2\left(S U B_{k}\right)>A N S \_3(P)$, which implies $A N S \_2\left(S U B_{k}\right)$ or $A N S_{-} \_\left(S U A_{k}\right)$ are not an optimal solution for $S U B_{k}$ or $S U A_{k}$. That is contradictory. Therefore, $t r \leq A N S 3(P)$. In summary, $A N S \_3(P)=\min \left(\max \left(A N S \_1\left(S U A_{k}\right), A N S \_2(S U\right.\right.$ $\left.\left.B_{k}\right)\right), 1 \leq k \leq \frac{n(n-3)}{2}$.

Lemma 5 For any number $i(1 \leq i \leq n-1)$, Assuming $\operatorname{br}(\langle i, k>)=z$ is the minimum in $\operatorname{gbr}(i)$, then ,the following relationship is established.

$\operatorname{br}(<i, i+1>) \geq \operatorname{br}(<i, i+2>) \geq \ldots \geq \operatorname{br}(<i, k>) \leq \operatorname{br}(<i, k+1>) \leq$ $\operatorname{br}(<i, k+2>) \leq \operatorname{br}(<i$,end $>)$

Proof. Assuming $\operatorname{br}(\langle i, k\rangle)=z$ is the minimum in $\operatorname{gbr}(i)$, According to the definition, when $\operatorname{br}(\langle i, k\rangle)$ takes a minimum value, there are the following two cases need to be discussed as follow:

Case 1. ANS_2 $\left.(\langle k+1, i-1\rangle) \leq A N S \_1(<i, k\rangle\right)=z$

Case 2. ANS_l $(<i, k>)<A N S \_2(<k+1, i-1>)=z$.

First of all, we discuss case1. We know that $A N S \_1(<i, k+1>) \geq A N S \_1(<i, k>)$ and $A N S \_2(<k+2, i-$ $1>) \leq A N S \_2(\langle k+2, i-1\rangle)$ by Lemma 4 , so we have $\operatorname{br}(<i, k>) \leq \operatorname{br}(<i, k+1>)$. According to Lemma 3, for any $m, \quad(k+1 \leq m \leq$ end -1$), \quad$ we have that $A N S \_1(<i, m+1>) \geq A N S \_1(<i, m>)$ and $A N S \_2(<m+2, i$ $1>) \leq A N S \_2(<m+1, i-1>)$, thus the formula is established as follow: $\operatorname{br}(<i, k>) \leq \operatorname{br}(<i, k+1>) \leq \operatorname{br}(<i, k+2>) \leq \operatorname{br}(<i$, end $>)$.

We have $\operatorname{br}(\langle i, k-1>) \geq \operatorname{br}(<i, k\rangle)$ because of the suppose that $\operatorname{br}(<i, k>)$ is minimum in $\operatorname{gbr}(i)$. If $\operatorname{br}(<i, k$ $1>)>\operatorname{br}(\langle i, k\rangle)$ holds, then we have that ANS_2 $(<k, i$ $1>)>A N S \_2(<i, k-1>)$ by lemma 3. According to the definition that $\operatorname{br}(<i, k-2\rangle)=\max \left\{A N S_{-} 1(<i, k\right.$ -

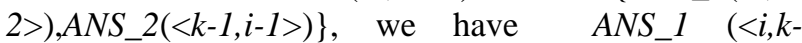
$\left.1>) \leq A N S \_1(<i, k-2\rangle\right) \quad$ and $\quad A N S \_2 \quad(<k-1, i$ $1>) \geq A N S \_2(\langle k, i-1\rangle)$ by lemma 4 , thus $\operatorname{br}(\langle i, k-2\rangle)=$ $\left.A N S \_2(<k-1, i-1\rangle\right) \geq A N S \_2(\langle k, i-1\rangle)=\operatorname{br}(\langle i, k-1\rangle)$. So, for any number $m(i+1 \leq m \leq k-3)$ that $\operatorname{br}(<i, m>) \geq \operatorname{br}(<i, m+1>)$ holds, so, we have $\operatorname{br}(\langle i, i+1\rangle) \geq \operatorname{br}(<i, i+2>) \geq \ldots \geq \operatorname{br}(<i, k\rangle)$. If $\operatorname{br}(\langle i, k-1\rangle)=$ br $(\langle i, k\rangle)$ holds and there is no number $m(i+1 \leq m \leq k-2)$ such that $\operatorname{br}(\langle i, m\rangle)\rangle \operatorname{br}(\langle i, k\rangle)$ holds, then we have $\operatorname{br}(<i, i+1>)=\operatorname{br}(<i, i+2>)=\ldots=\operatorname{br}(<i, k>)$ by lemma 3 . If there is a number $m(i+1 \leq m \leq k-2)$ such that $\operatorname{br}(\langle i, m\rangle)>\operatorname{br}(\langle i, k\rangle)$ holds. According to the previous discussion, we can know that $\operatorname{br}(<i, i+1>) \geq \operatorname{br}(<i, i+2>) \geq \operatorname{br}(<i, m>) \quad=\ldots=\quad \operatorname{br}(<i$, $k\rangle)=\operatorname{br}(\langle i, k+1\rangle)$. Proposition is proved. The proof process for Case 2 is similar to Case 1. Thus, Lemma 5 is hold.

We can now give the result of this paper.

Theorem 1 Given a set $S$ of $n$ points in convex position, we can compute in $O\left(n^{2} \log ^{3} n\right)$ time the minimum radius $r$ such that union of three disks of radius $r$ covers $S$.

Proof. According to Lemma 4 and its related definitions, we can get the formula as follow. $A N S \_3(S)=\min \{\operatorname{gbr}(1), \operatorname{gbr}(2), \ldots, \operatorname{gbr}(n)\}$. For any number $i, \operatorname{gbr}(i)$ is a monotonic sequence according to Lemma 5. We find the minimum value in $\operatorname{gbr}(i)$ by using binary search, which uses $O(\log n)$ time. The planar 1-center problem can be solved in $O(n)$ time[3], and the planar 2center can be solved in $O\left(n \log ^{2} n\right)$ [9].So, for any number $i$, we can compute in $O\left(n \log ^{3} n\right)$ time algorithm time the minimum value in sequence $\operatorname{gbr}(i)$.Using $O(n)$ time to traverse all elements in $A N S \_3(S)$. Thus we can compute ANS_3(S) in $O\left(n^{2} \log ^{3} n\right)$ time .

Our algorithm steps are described as follows

Algorithm ThreeCenter $(V)$

Input: $V$ (The set of vertices of an arbitrary convex polygon $P$ )。

Output: Returns the ans of the 3-center problem of convex position points

$1 \quad V \leftarrow$ ConvexHull $(V)[10]$

2 According to the $X$-coordinates, find the one with the smallest $X$-coordinate value in the sequence $v$, and use this as a starting point to arrange $v$ in the reverse direction, and then start the numbering from 0 .Then Get the sequence, $v_{0}, v_{1}, \ldots, v_{n-1}$

3 Generate a empty lists global_l

4 for $i \leftarrow 0$ to $n$

Add binarySearch $(i, V)$ to the global_l

6 Assign the minimum value in global_l to ans 
7 return ans;

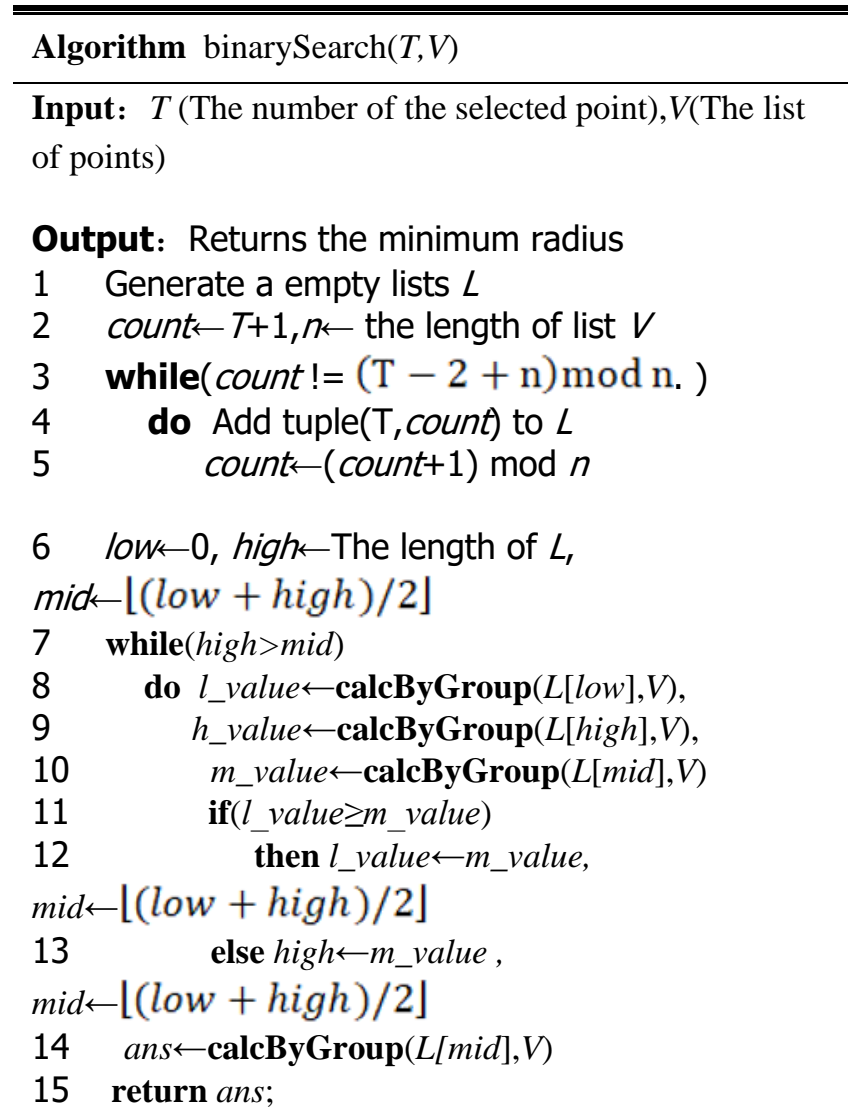

\begin{tabular}{l}
\hline \hline Algorithm calcByGroup $(T, V)$ \\
\hline Input: $T$ (Two dimensional tuple), (The list of points)
\end{tabular}

Output: Returns radius

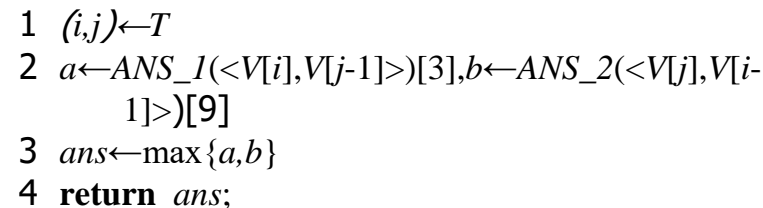

\section{Conclusions}

In this paper, we first introduced the planar $k$-center problem and the relevant problems which have been studied widely. Then we presented an $O\left(n^{2} \log ^{3} n\right)$ time algorithm to solved the restricted planar 3-center problem. Our results are mainly obtained by dividing the 3 -center problem into a 1-center problem and a 2-center problem. Our algorithm is simple and easy to implement. It is an interesting work to develop a faster algorithm to solve the planar 3-center problem for arbitrarily given points.

\section{Acknowledgement}

This work was partially supported by National Natural Science Foundation of China under grant 61702242.

\section{References}

1. Megiddo, N., Supowit, K.: On the complexity of some common geometric location problems. SIAM J. Comput. 13, 1182-1196 (1984)

2. Hwang, R.Z., Lee, R.C.T., Chang, R.C.: The slab dividing approach to the euclidean P-center problem. Algorithmica 9, 1-22 (1993)

3. Chazelle, B., Matou sek, J.: On linear-time deterministic algorithms for optimization problems in fixed dimension. J. Algorithms 21, 579-597 (1996)

4. Agarwal, P.K., Sharir, M., Welzl, E.: The discrete 2-center problem. Discret. Comput. Geom. 20, 287-305 (1998)

5. Eppstein, D.: Faster construction of planar twocenters. In: Proceedings of 8th ACM-SIAM Symposium on Discrete Algorithms, pp. 131-138 (1997)

6. Chan, T.M.: More planar two-center algorithms. Comput. Geom. Theor. Appl. 13, 189-198 (1999)

7. Dyer, M.E.: On a multidimensional search technique and its application to the Euclidean onecenter problem. SIAM J. Comput. 15, 725-738 (1986)

8. Kim, S.K., Shin, C.-S.: Efficient algorithms for two-center problems for a convex polygon. In: Du, D.-Z.-Z., Eades, P., Estivill-Castro, V., Lin, X., Sharma, A. (eds.) COCOON 2000. LNCS, vol. 1858, pp. 299-309. Springer, Heidelberg (2000).

9. Tan X, Jiang B. Simple $\mathrm{O}\left(n \log ^{2} n\right)$ Algorithms for the Planar 2-Center Problem[C], International Computing and Combinatorics Conference. Springer, Cham, 2017: 481-491.

10. Graham R L. An efficient algorith for determining the convex hull of a finite planar set. Information Processing Letters, 1972, 1(4):132-133. 